\title{
Surgical treatment of femoroacetabular impingement in a patient with Stickler syndrome: a case report
}

\author{
Yiou Wang, Yanyan Bian, Xi Chen, Wenwei Qian \\ Department of Orthopedic Surgery, Peking Union Medical College Hospital, Chinese Academy of Medical Sciences and Peking Union Medical \\ College, Beijing, China \\ Correspondence to: Wenwei Qian, MD. Department of Orthopedic Surgery, Peking Union Medical College Hospital, Chinese Academy of Medical \\ Sciences and Peking Union Medical College, Beijing 100730, China. Email: qianww007@163.com.
}

\begin{abstract}
Stickler syndrome is a multisystem connective tissue disorder caused by mutations in collagen genes that can present with craniofacial, ocular, audial, or skeletal abnormalities. Here, we report on a male patient with a COL2A1 missense mutation (c.647G>A; p.Gly216Asp). He complained of an out-toeing gait and restricted hip mobility. Radiographs showed broad and elongated femoral necks with coxa valga. An alpha angle of $119^{\circ}$ and $96^{\circ}$ for his left and right femur, respectively, and almost no femoral head-neck offset, suggested a femoroacetabular impingement. Considering the patient's unwillingness to receive a total hip replacement for his Tönnis grade 2 hips, we intended to establish impingement-free hips by causing minimizing trauma. Therefore, we performed an osteochondroplasty of femoral head-neck junction and gluteal muscle release without correcting coxa valgus through the surgical hip dislocation approach. The range of motion of his hips improved as the surgery restored the femoral head-neck offset. However, the patient experienced a sense of lower limb length disparity and hip instability, which might be caused by his uncorrected proximal femoral deformity. This case presents the previously unreported phenotypic features of a COL2A1 mutation G216D. Orthopedic surgeons should consider genetic disorders when confronting atypical abnormalities. Moreover, the primary deformity should be corrected in hip preservation surgeries. Insufficient deformity correction might contribute to unsatisfactory surgical outcomes.
\end{abstract}

Keywords: Stickler syndrome; COL2A1 G216D mutation; femoroacetabular impingement; surgical hip dislocation; case report

Submitted Sep 10, 2021. Accepted for publication Jan 14, 2022.

doi: $10.21037 / \mathrm{atm}-21-4784$

View this article at: https://dx.doi.org/10.21037/atm-21-4784

\section{Introduction}

Stickler syndrome is an inherited connective tissue disorder that was first described in 1965 (1). It is characterized by premature osteoarthritis, craniofacial defects, ocular abnormalities, and hearing impairment. Pathogenic variants in one of six genes (COL2A1, COL11A1, COL11A2, COL9A1, COL9A2, COL9A3) which affect collagen production, mainly collagen type II, contribute to Stickler syndrome (2). Hip abnormalities are commonly observed, but present with a broad spectrum of manifestations, eliciting challenges for diagnosis. An alteration in epiphysis may lead to a femoroacetabular impingement causing hip pain and a limited range of motion (3). Here we report on a male with a significant deformity in his hips caused by the COL2A1 mutation (p.Gly216Asp). The reason for publishing this case report is not simply to present the phenotypic features in relation to this previously unreported mutation, but also to highlight potential issues to the reader when treating such deformities. We present the following article in accordance with the CARE reporting checklist (available at https://atm.amegroups.com/article/ view/10.21037/atm-21-4784/rc).

\section{Case presentation}

All procedures performed in this study were in accordance 
with the ethical standards of the institutional and/or national research committee(s) and with the Helsinki Declaration (as revised in 2013). Written informed consent was obtained from the patient for publication of this case report and accompanying images. A copy of the written consent is available for review by the editorial office of this journal.

\section{Clinical features}

A 22-year-old man presented with a limp and limitation of motion. The limp, with an out-toeing gait, began when he was 3 years old, and his limitation of motion had worsened as he became order. However, he reported only mild pain in his lower back and groin region. He had undergone a cleft palate repair at 2 years old and had developed myopia and astigmatism before school age.

Physical examination revealed a marfanoid habitus with a height of $196 \mathrm{~cm}$ and a weight of $98 \mathrm{~kg}$. His head examination showed a flattened nasal bridge and micrognathia. Both hips were almost fixed in an external rotational position (left $30^{\circ}$, right $45^{\circ}$ ). The range of motion was limited to $60^{\circ}$ in flexion, and $10^{\circ}$ in abduction and adduction. Owing to his long-term limitation of motion, he developed an out-toeing gait with thigh abduction similar to Charlie Chaplin in his movies. Because of his restricted external rotated hip, when seated with his knee flexed, his leg crossed, the left foot on the right and the right foot on the left.

All laboratory tests, including rheumatology and virology serology, revealed findings within normal ranges. Pure tone audiometry showed sensorineural hearing impairment in the high-frequency range. Vitreous anomaly and retinopathy were not found in the ophthalmic examination. With the exception of severe fatty liver, an ultrasound scan revealed no obvious other abnormalities in his heart, kidney, or pancreas.

Anteroposterior (AP) view radiographs revealed an aspheric femoral head, elongated and broad femoral neck with coxa valga, and indistinctness and flattening of the head-neck junction (Figure 1A). With narrowing joint space, both hips were classified as Tönnis grade 2. As for femoral morphology, the caput-collum diaphyseal (CCD) angle measured $154^{\circ}$ and $157^{\circ}$ (left and right respectively). The lateral view (Figure 1B,1C) revealed an alpha angle of $119^{\circ}$ for the left femur, $96^{\circ}$ for the right femur, and nearly no offset for the femoral head-neck junction. As for acetabular coverage, the Wiberg center edge angle was $31^{\circ}$ for the left hip and $34^{\circ}$ for the right hip. These findings suggested a cam-type femoroacetabular impingement.
Spinal radiographs showed loss of lumbar lordosis without scoliosis and endplate abnormalities. There was no sign of arthropathy in the knees, hands, or feet.

\section{Diagnosis}

The patient's mother had similar but less severe hip deformities and myopia. No similar abnormalities were revealed in his other relatives. DNA was extracted from the peripheral blood of the patient and his mother, and a wholeexome sequencing analysis was performed. A missense mutation c.647G>A (p.Gly216Asp) in the COL2A1 gene was found. This mutation was previously reported in a patient with a clinical diagnosis of Stickler syndrome, but the article did not provide the specific phenotypic features of this patient (4). According to all the findings above, our patient was, however, shown to fulfill the clinical diagnostic criteria for Stickler syndrome (5).

\section{Treatment}

According to the patient's clinical and radiographic manifestations, we discussed a total hip arthroplasty with him and his parents. They refused replacement and requested hip preservation treatment. We, therefore decided to perform an osteochondroplasty of the femoral head-neck junction, combined with gluteal muscle release (6) to improve the restricted motion of the hips. Considering that the extensive lesion involved the posterior aspect of head-neck conjunction, it would have been difficult to carry out an arthroscopic resection of the entire cam deformity. Hence, we chose the open surgical hip dislocation approach described by Ganz et al. (7).

Before dislocation, the patient's hips were moved through an arc of motion to localize the areas of impingement. The femoral head-neck junction was then recontoured with a rongeur and round bur to remove the impingement bump. Considering the patient's tall stature and weight, we did not remove excessive bone bulk so as not to risk femoral neck fracture. The tightness of the tensor fasciae latae, gluteus maximus, gluteus medius, and gluteus minimus was sequentially released, and examination indicated that both hips returned to an appropriate range of motion.

The out-toeing posture was corrected to a neutral position, and the motion of the hips improved $\left(100^{\circ}\right.$ in flexion; $10^{\circ} / 40^{\circ}$ internal/external rotation) postoperatively. Postoperative radiographs (Figure $2 A-2 C$ ) revealed the restoration of femoral head neck morphology. The alpha 

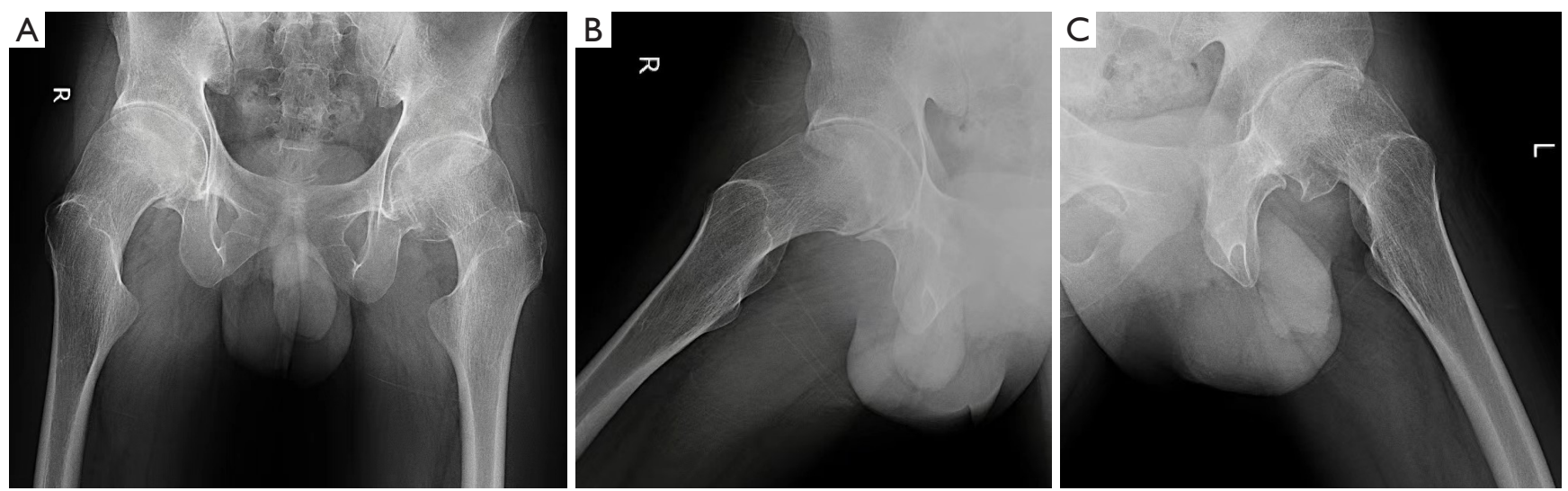

Figure 1 Preoperative (A) AP pelvic view, (B) and (C) the lateral view radiographs presented the hypertrophy of the femoral neck caused indistinctness of the femoral head-neck junction. AP, anteroposterior.
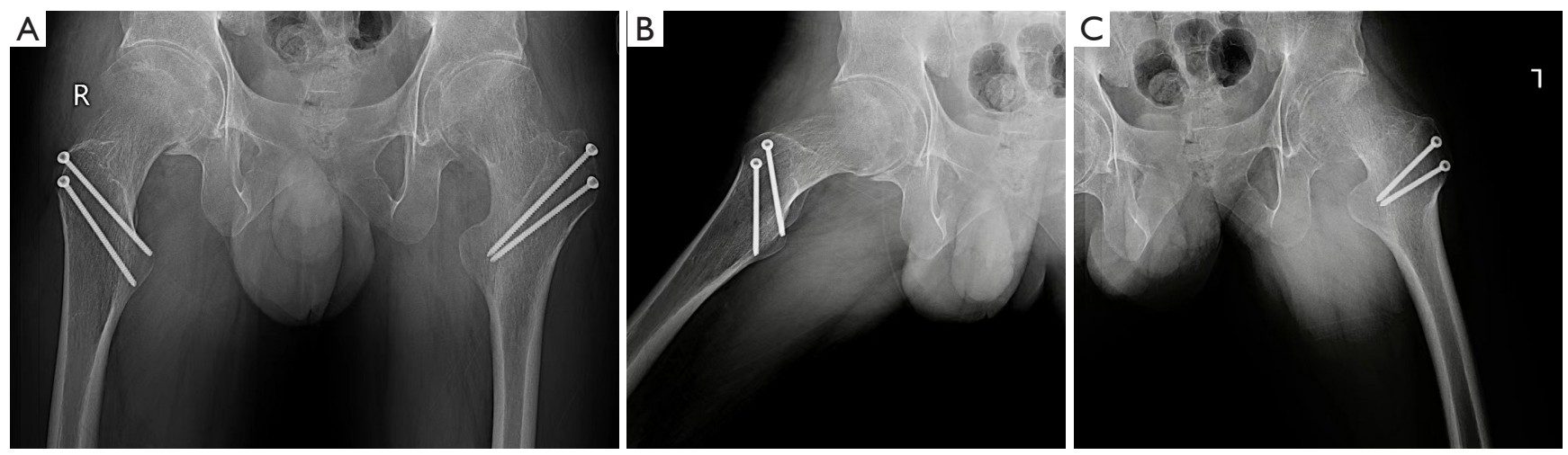

Figure 2 Postoperative (A) AP pelvic view, (B) and (C) lateral view radiographs revealed the restoration of femoral head-neck junction morphology. AP, anteroposterior.

angle has been reduced by $46^{\circ}$ for the left and $43^{\circ}$ for the right femur, and a 7.4 and $8.4 \mathrm{~mm}$ of head-neck junction offset was produced for left and right femur, respectively. Nevertheless, the patient experienced a sense of lower limb length disparity and hip instability, without pain, postoperatively. After a year of rehabilitation, his discomforts had not ameliorated significantly. However, he continued to report an absence of pain, and radiographs revealed no avascular necrosis of the femoral head. The patient then considered total hip arthroplasty.

\section{Discussion}

This is the first report of the phenotypic features of a patient with a COL2A1 mutation G216D. G216D results in the substitution of a glycine, which hampers the folding of the procollagen chains into a triple helix structure, contributing to type II collagen disorder. The COL2A1 mutation usually leads to shorter stature (8). However, our patient exhibited a tall stature and marfanoid habitus and, with the exception of his hips, had no severe musculoskeletal deformity, which is uncommon. This supports the finding that severe and short stature phenotypes do not occur with the $\mathrm{N}$-terminal to the glycine at position $303(4,9)$. Interestingly, in anecdotal case reports, we noted that patients with a COL $2 A 1$ mutation in tall stature always have a concomitant coxa valga (10-12). However, their relationship is unclear.

Hip abnormalities are common but various in Stickler syndrome. A study of 51 patients with hip radiographs revealed that $63 \%$ of patients reported chronic hip pain, and the proportion rose to $79 \%$ in adulthood (13). Specific hip manifestations include protrusio acetabuli, coxa valga, slipped epiphysis, Legg-Perthes-like disease, and chondrolysis. Al Kaissi suggested that the COL2A1 
mutation impacts early bone formation and contributes to poor anatomical arrangements at the epimetaphyseal junction (11). Literature on coxa valga is common, but scarce with regard to patients with broad femoral necks.

Orthopedic surgeons may need to consider genetic syndromes, particularly with atypical presentations, such as those of the Stickler syndrome reported here. Early and correct diagnosis of these diseases can facilitate the evaluation and management of other systemic manifestations.

Considering that the patient already had signs of osteoarthritis, our goals were as follows: (I) to establish impingement-free hips in most daily activities; (II) to correct the abnormal posture and gait; and (III) to minimize surgical trauma. Therefore, we chose to solely remove the impingement bumps in the femoral head-neck conjunction, combined with gluteal release without osteotomy to correct the coxa valga through the surgical hip dislocation approach. However, the surgical outcome was not satisfactory. This was because the leg length discrepancy came from the absolute length of the femur, which was confirmed in the full-length radiograph. However, the preoperative different femur adduction and external rotation, slight habitual knee flexion, and pelvic tilt, compensated for this inequality, which manifested after the out-toeing posture was corrected and the unbalanced tight gluteus was released. Surgeons need to be aware of differences in the subtrochanteric length in patients with proximal femur deformity (14). In addition, differences in femoral morphology and rotation could cause a lower limp disparity measurement error when using greater or lesser trochanter in the pelvic AP radiograph. For these patients, a leg-long radiograph or computed tomography from hip to knee is recommended (15). Hip instability might be attributed to valgus hip and gluteal weakness after release. Coxa valga and concomitant decreased lateral offset may result in posterior impingement and hip dynamic instability (16). We hypothesize that the unstable valgus hips attained temporal stability through femoral neck hypertrophy and gluteal contracture during his growth, and our surgery broke this stability.

This unsuccessful surgery suggested that hip preservation surgery should address the primary deformity rather than secondary change. In addition, insufficient deformity correction is also the primary reason for postoperative pain (17).

\section{Acknowledgments}

Funding: This study was supported by grants from the
Beijing Municipal Natural Science Foundation (No. 7192173), the Peking Union Medical College Hospital Foundation (No. ZC201904183), and the National Natural Science Foundation of China (No. 81972046).

\section{Footnote}

Reporting Checklist: The authors have completed the CARE reporting checklist. Available at https://atm.amegroups. com/article/view/10.21037/atm-21-4784/rc

Conflicts of Interest: All authors have completed the ICMJE uniform disclosure form (available at https://atm. amegroups.com/article/view/10.21037/atm-21-4784/coif). The authors have no conflicts of interest to declare.

Ethical Statement: The authors are accountable for all aspects of the work in ensuring that questions related to the accuracy or integrity of any part of the work are appropriately investigated and resolved. All procedures performed in this study were in accordance with the ethical standards of the institutional and/or national research committee(s) and with the Helsinki Declaration (as revised in 2013). Written informed consent was obtained from the patient for publication of this case report and accompanying images. A copy of the written consent is available for review by the editorial office of this journal.

Open Access Statement: This is an Open Access article distributed in accordance with the Creative Commons Attribution-NonCommercial-NoDerivs 4.0 International License (CC BY-NC-ND 4.0), which permits the noncommercial replication and distribution of the article with the strict proviso that no changes or edits are made and the original work is properly cited (including links to both the formal publication through the relevant DOI and the license). See: https://creativecommons.org/licenses/by-nc-nd/4.0/.

\section{References}

1. Stickler GB, Belau PG, Farrell FJ, et al. Hereditary progressive arthro-ophthalmopathy. Mayo Clin Proc 1965;40:433-55.

2. Robin NH, Moran RT, Ala-Kokko L. Stickler Syndrome. In: Adam MP, Ardinger HH, Pagon RA, et al. editors. GeneReviews ${ }^{\circledR}$. Seattle: University of Washington, 2000.

3. Morris WZ, Li RT, Liu RW, et al. Origin of Cam Morphology in Femoroacetabular Impingement. Am J 
Sports Med 2018;46:478-86.

4. Hoornaert KP, Vereecke I, Dewinter C, et al. Stickler syndrome caused by COL2A1 mutations: genotypephenotype correlation in a series of 100 patients. Eur J Hum Genet 2010;18:872-80.

5. Rose PS, Levy HP, Liberfarb RM, et al. Stickler syndrome: clinical characteristics and diagnostic criteria. Am J Med Genet A 2005;138A:199-207.

6. Alves K, Katz JN, Sabatini CS. Gluteal Fibrosis and Its Surgical Treatment. J Bone Joint Surg Am 2019;101:361-8.

7. Ganz R, Gill TJ, Gautier E, et al. Surgical dislocation of the adult hip a technique with full access to the femoral head and acetabulum without the risk of avascular necrosis. J Bone Joint Surg Br 2001;83:1119-24.

8. Terhal PA, van Dommelen P, Le Merrer M, et al. Mutation-based growth charts for SEDC and other COL2A1 related dysplasias. Am J Med Genet C Semin Med Genet 2012;160C:205-16.

9. Terhal PA, Nievelstein RJ, Verver EJ, et al. A study of the clinical and radiological features in a cohort of 93 patients with a COL2A1 mutation causing spondyloepiphyseal dysplasia congenita or a related phenotype. Am J Med Genet A 2015;167A:461-75.

10. Carlson KM, Yamaga KM, Reinker KA, et al. Precocious osteoarthritis in a family with recurrent COL2A1

Cite this article as: Wang Y, Bian Y, Chen X, Qian W. Surgical treatment of femoroacetabular impingement in a patient with Stickler syndrome: a case report. Ann Transl Med 2022;10(8):495. doi: $10.21037 /$ atm-21-4784 mutation. J Rheumatol 2006;33:1133-6.

11. Al Kaissi A, Roschger P, Nawrot-Wawrzyniak K, et al. Evidence of reduced bone turnover and disturbed mineralization process in a boy with Stickler syndrome. Calcif Tissue Int 2010;86:126-31.

12. Al Kaissi A, Klaushofer K, Grill F. Osteochondritis dissecans and Osgood Schlatter disease in a family with Stickler syndrome. Pediatr Rheumatol Online J 2009;7:4.

13. Rose PS, Ahn NU, Levy HP, et al. The hip in Stickler syndrome. J Pediatr Orthop 2001;21:657-63.

14. Tolk JJ, Eastwood DM, Hashemi-Nejad A. Leg length discrepancy in patients with Perthes' disease : a note of caution for the arthroplasty surgeon. Bone Joint J 2021;103-B:1736-41.

15. Tamura K, Takao M, Hamada H, et al. Femoral morphology asymmetry in hip dysplasia makes radiological leg length measurement inaccurate. Bone Joint J 2019;101-B:297-302.

16. Siebenrock KA, Steppacher SD, Haefeli PC, et al. Valgus hip with high antetorsion causes pain through posterior extraarticular FAI. Clin Orthop Relat Res 2013;471:3774-80.

17. Hanke MS, Lerch TD, Schmaranzer F, et al. Complications of hip preserving surgery. EFORT Open Rev 2021;6:472-86. 